\title{
Hunting the Ethical State. The Benkadi Movement of Côte-d'Ivoire.
}

Chicago, University of Chicago Press, 2011, 312 p., bibl.

\section{Agnès Kedzierska Manzon}

\section{(2) OpenEdition}

\section{Journals}

\section{Édition électronique}

URL : http://journals.openedition.org/etudesafricaines/17863

DOI : 10.4000/etudesafricaines. 17863

ISSN : 1777-5353

\section{Éditeur}

Éditions de l'EHESS

Édition imprimée

Date de publication : 3 octobre 2014

ISSN : 0008-0055

\section{Référence électronique}

Agnès Kedzierska Manzon, « Hunting the Ethical State. The Benkadi Movement of Côte-d'Ivoire. »,

Cahiers d'études africaines [En ligne], 215 | 2014, mis en ligne le 02 octobre 2016, consulté le 24

septembre 2020. URL : http://journals.openedition.org/etudesafricaines/17863 ; DOI : https://doi.org/

10.4000/etudesafricaines. 17863

Ce document a été généré automatiquement le 24 septembre 2020

(C) Cahiers d'Études africaines 


\title{
Hunting the Ethical State. The Benkadi Movement of Côte-d'Ivoire.
}

Chicago, University of Chicago Press, 2011, 312 p., bibl.

\author{
Agnès Kedzierska Manzon
}

\section{HELLWEG, Joseph. - Hunting the Ethical State. The Benkadi Movement of Côte-d'Ivoire. Chicago, University of Chicago Press, 2011, 312 p., bibl.}

1 La Côte-d'Ivoire, qui vient de traverser dix années de crise nationale, de quasi-guerre civile et de violences intermittentes, vit encore un moment de transition après le rétablissement de la démocratie. L'anthropologue américain Joseph Hellweg consacre une belle monographie au mouvement Benkadi, né dans le nord de la Côte-d'Ivoire, au début des années 1990. À l'époque, lorsque la police ivoirienne s'est révélée incapable de juguler une vague de criminalité généralisée - marquée, notamment, par les exactions des fameux " coupeurs de route ", la version ouest-africaine des bandits de grands chemins -, des maîtres chasseurs appelés dozo endossèrent le rôle de policiers volontaires. Aux yeux d'une grande partie de la population, ces chasseurs semblaient capables de redresser les travers d'une société en crise, en raison d'une initiation fondée sur des valeurs éthiques allant bien au-delà de l'art de la chasse. Lorsque l'on parle de dozo (dérivé du malinké donso), il ne s'agit pas seulement de spécialistes de la chasse. D'ailleurs, le gibier s'étant catastrophiquement raréfié en Côte-d'Ivoire, la chasse est, de fait, devenue un peu folklorique. De ce point de vue, la transformation des sociétés de chasseurs en mouvement sécuritaire à vocation nationale, peut apparaître comme une astucieuse adaptation au changement.

2 Tout en se réclamant de l'islam, les dozo ivoiriens font des sacrifices à leur génie tutélaire, Manimory, afin de fusionner avec la nature. Grâce à ce patronage, ils ont la réputation d'être omniscients et dotés de pouvoirs thaumaturgiques. Hellweg commence par analyser les tropes qui structurent la conception dozo de la chasse et qui éclairent les relations de Manimory avec la brousse. Il étudie ensuite les récits oraux 
faisant référence à ce personnage et d'autres héros chasseurs. Les chants et les incantations des chasseurs l'aident à montrer comment la mimesis entre Manimory et la forêt sert de trope grâce auquel les dozo organisent leur relation à l'islam et, par extension à l'État ivoirien (voir notamment p. 104). La région d'Odienné où Hellweg a mené l'essentiel de ses enquêtes est depuis longtemps massivement islamisée, mais cela n'empêche pas de nombreuses personnes de pratiquer la divination, les danses de masques et les sacrifices aux ancêtres ou à d'autres entités invisibles. Les règles initiatiques des dozo lient leurs succès à la chasse, à leur conduite morale et à leurs règles de vie rigoureuses. Les dozo font en effet le serment à Manimory de ne pas voler, de ne pas commettre l'adultère, de ne pas mentir, ni de trahir d'autres chasseurs. Les dozo appelèrent leur mouvement Benkadi, ce qui en jula signifie «la concorde est douce ». Ils surent utiliser habilement la symbolique ancienne liée à la chasse, aux savoirs occultes et à une profonde connaissance de la nature pour se transformer en sorte d'agents supplétifs d'un État défaillant. Les agents de Benkadi organisèrent des patrouilles de sécurité à travers tout le pays, arrêtèrent des criminels et restituèrent à leurs propriétaires pas mal de biens volés. Le mouvement grossit au point de comprendre des dizaines d'hommes armés. Selon l'auteur - et l'hypothèse est convaincante - les dozo conçurent leur rôle de policiers dans des termes sacrificiels identiques à ceux qui président à leurs activités de chasseurs. Le prestige des chasseurs dépasse d'ailleurs largement le nord de la Côte-d'Ivoire. En Guinée, le président Lansana Conté a mobilisé les chasseurs à plusieurs reprises. Au Mali et au Burkina Faso, certaines personnalités politiques s'appuyèrent sur le prestige des chasseurs pour susciter l'intérêt de bailleurs de fonds férus d'authenticité culturelle. Mais en Côted'Ivoire, le mouvement Benkadi prit une telle ampleur que certains officiels s'en inquiétèrent. Ils en vinrent à considérer ce mouvement comme une sorte de milice favorable à $\mathrm{Al}$-assane Ouatara, ancien Premier ministre d'Houphouët-Boigny devenu président du pays après la longue crise résultant du refus de Laurent Gbagbo de reconnaître sa défaite électorale. À l'époque, Ouatara était souvent perçu - c'est une image qu'il s'efforce aujourd'hui de transcender - comme homme du Nord musulman, par opposition à un Sud en majorité chrétien. Quoi qu'il en soit, le gouvernement ivoirien commença à réprimer le mouvement Benkadi, puis l'interdit carrément en 1998. Hellweg relève une ironie de l'histoire qui nuance les clivages politiques trop simples. En effet, lorsque Benkadi apparut la première fois en tant que milice, en 1995, c'était à la requête du PDCI (Parti démocratique de Côte-d'Ivoire) de Konan Bedié et non du RDR (Rassemblement des républicains de Côte-d'Ivoire) d'Al-assane Ouatara (p. 44). Par ailleurs, l'auteur fait justement remarquer qu'après son coup d'État à la fin de 1999, le général Guëi invita les dozo à Abidjan pour garder les places publiques et les centres commerciaux, ainsi que le palais présidentiel; ce qui ne l'empêcha pas de faire approuver par referendum une nouvelle constitution qui rendait Al-assane Ouatara inéligible. Bien sûr, les rituels dozo s'enracinent dans le Mandé, mais ils montrent une capacité remarquable à intégrer des gens de langues et d'affiliations religieuses différentes (p. 69). Après l'assassinat du général Guëi et l'arrivée au pouvoir de Laurent Gbagbo, les associations de chasseurs connurent une nouvelle éclipse, Gbagbo ayant ses propres milices informelles: les tristement célèbres «jeunes patriotes ». Mais en 2002, lorsque la rébellion éclata dans le nord du pays, les dozo réapparurent.

3 Hellweg a le mérite de replacer le redéploiement des chasseurs dans son contexte sociologique, tout en fondant son analyse sur une solide enquête de terrain. La proximité - le face à face, le coude à coude - reste, à l'évidence, la plus efficace des 
méthodes d'enquête. Même si Hellweg n'est pas un chasseur pratiquant, il a pu en approcher le statut en participant intensément à la vie de la société des chasseurs. Il a même poussé le zèle jusqu'à travailler aux champs avec ses hôtes, ce qui n'est pas une mince affaire. Comment un mouvement comme Benkadi a-t-il pu se développer? En février 1994, la dévaluation du franc CFA, une mesure censée stimuler les exportations, déboucha sur une aggravation de la pauvreté, qui à son tour stimula la criminalité. Les ajustements structurels imposés par les organisations financières internationales laissèrent l'État ivoirien avec si peu de ressources qu'il s'avéra incapable de jouer son rôle de gardien de la loi et d'incarner l'ordre public. Ces déficiences de l'État ouvrirent la porte à des mouvements spontanés comme Benkadi, ainsi qu'à la prolifération d'oNG, qui, peu à peu, se substituèrent aux services publics. Pour compléter leur maigre solde, les policiers recoururent au racket et monnayèrent leurs services, tandis que des compagnies de sécurité privées commençaient à louer leurs services aux citoyens les plus fortunés. Quant à ceux qui n'avaient pas les moyens d'y recourir, ils s'organisèrent parfois en groupes de vigiles volontaires. La passivité de l'État face à la criminalité et sa volonté de sous-traiter au maximum à des opérateurs privés a fait de Benkadi à la fois le produit de l'État et un rival de l'État. Hellweg emploie le concept de nemesis (p. 30), mais longtemps avant la crise de l'État postcolonial, les destinées de chasseurs et de l'État étaient déjà entremêlées, même si l'auteur ne s'appesantit pas sur ce sujet. Joseph Hellweg ne cède jamais à l'hagiographie, qui guette toujours l'ethnographe soucieux de partager le mode de vie de ses hôtes. Il reconnaît que bien qu'ils se réclamassent d'un grand sens éthique, les dozo furent loin d'être toujours irréprochables; ce qui rejoint les observations de Thomas Basset. Plusieurs dérapages portèrent sérieusement atteinte à leur réputation de rigueur, de sagesse et d'impartialité. Dans un pays où les forces de l'ordre appliquaient la loi comme ils l'entendaient, les dozo de Benkadi, en les imitant, firent pareil, même si on est encore loin des dérives des Kamajor, les milices de chasseurs de Sierra Leone, ou des Cobra, Ninja et Coxoye du Congo : « Ils s'identifièrent, écrit Hellweg, avec un pouvoir situé au-dessus des lois » (p. 179).

L'anthropologue américain note que, lors des contentieux, dans un curieux renversement de rôles, un bureaucrate dozo, donc officieux, se chargeait de recueillir consciencieusement les dépositions et les données administratives des citoyens. Ce phénomène n'est pas nouveau et l'auteur aurait pu noter que ce type de mimétisme était déjà à l'œuvre dans la vie associative, notamment dans les fameuses goumbés qui sont à la fois sociétés d'entraide, de fêtes et de danses. La lecture d'un règlement imitant ceux de l'administration servait d'ailleurs de narration au film de Jean Rouch tourné en 1965, La Goumbé des jeunes noceurs.

5 Quelques critiques mineures qui ne portent pas atteinte au remarquable travail de Hellweg peuvent être formulées: l'excès de notes en fin de volume complique inutilement la lecture, l'accord du pluriel pour les mots malinké est contestable (danguns, dozos, etc.) et la trop scrupuleuse mention des références savantes donne parfois l'impression de judiciariser le texte ethnographique. Ces remarques ne pèsent guère au regard d'un travail qui allie la rigueur ethnographique à la réflexion théorique et qui présente en outre l'originalité d'accorder une place à l'esthétique, prise dans un sens voisin de celui proposé par Howard Morphy: il s'agit d'une hiérarchie de préférences pour satisfaire des désirs sensuels ou, en d'autres mots, d'un déploiement inventif de signes et de symboles dérivés d'une expérience sensorielle (p. 108). Il ne veut toutefois pas essentialiser l'esthétique comme une catégorie transculturelle, car, 
demeurant sur ce point «boasien», il souligne la relativité de tous les systèmes de classification. En conclusion, disons que cet ouvrage captivera tous les lecteurs soucieux de comprendre l'Afrique contemporaine, toujours en proie au travail d'adaptation d'héritages toujours sommés de répondre aux questions du présent.

7 Les chasseurs occupent une place privilégiée dans l'imaginaire mandingue. Considérés comme de véritables experts religieux, ils exerçaient par le passé et exercent aujourd'hui encore de multiples fonctions - de la protection des communautés au traitement des maladies en passant par la fondation des villages, sans parler de l'activité cynégétique. Pourtant, les publications à leur sujet se limitent aux actes d'un colloque, à quelques éditions des chants et à une quinzaine d'articles relativement récents. Hunting the Ethical State : the Benkadi Movement of Côte-d'Ivoire de Joseph Hellweg, est, avec le livre de Youssouf Tata Cissé (1994) ${ }^{25}$, le seul ouvrage anthropologique (et le premier en anglais) qui leur soit entièrement consacré. Fruit d'un travail de terrain mené par l'auteur depuis 1993, il aborde la tradition cynégétique mandingue dans toute sa complexité. Si la définition qu'il en donne (« un ethos rituel») étonne en ce qu'elle semble imposer au phénomène étudié une grille de lecture morale ${ }^{26}$, elle met en valeur la permanence d'une certaine logique symbolique dont la chasse constituait historiquement le domaine privilégié mais non exclusif de l'actualisation et qui s'étend aujourd'hui à d'autres domaines. Cette définition permet à l'auteur d'interpréter les mouvements contemporains des chasseurs - nombreux dans les pays ouest-africains non en rupture mais en continuité avec le passé.

rage commence par l'examen de la situation sociopolitique ivoirienne des années 1990, contexte nécessaire pour appréhender la naissance, le développement et l'effondrement du mouvement Benkadi dont Hellweg décrit la courte vie au travers d'une narration à la première personne. Les exemples puisés dans ses expériences fonctionnent comme des points d'ancrage des conclusions plus générales. La description des rapports domestiques lui sert de préambule pour l'analyse de l'univers cynégétique. Ce dernier s'enracine selon lui dans la même logique que la sphère domestique, régie par le principe de la réciprocité généralisée. L'initiation du chasseur en serait un parfait symbole. Tout comme le mariage ou le rituel de l'attribution du nom à un nourrisson, cette initiation crée un lien qui unit, dans ce cas précis, l'initié à la forêt d'une part, et d'autre part ce même initié à la communauté humaine (celle des chasseurs et, par extension, villageoise). Ce lien s'actualise à l'occasion d'autres offrandes rituelles des chasseurs mais également au cours de leur activité en brousse qu'Hellweg identifie à une forme de sacrifice, du fait de la distribution de viande qui en résulte et qui se déroule selon des codes stricts. C'est pourquoi la chasse et le rituel ces véritables "techniques de soi» (Hellweg 2009)27 - s'apparentent pour lui aux interventions anticriminelles des chasseurs, en ce qu'elles visent toutes à restaurer ou à soutenir un cycle de réciprocité temporairement rompu ou menacée ${ }^{28}$.

Pour approfondir cette hypothèse, Hellweg analyse les versions ivoiriennes des mythes fondateurs de l'organisation des chasseurs, ce qui l'amène à s'intéresser à la mimesis. Il rapporte que la capacité d'imiter le milieu environnant y est attribuée au premier héros-chasseur qui, après avoir accompli des exploits cynégétiques extraordinaires, disparaît en brousse sans laisser de traces, c'est-à-dire en s'assimilant totalement à cet espace. Par cette fin que Hellweg qualifie d'auto-sacrificielle, le héros lègue ses connaissances aux autres chasseurs. Ceux-ci, à travers l'initiation, réactualisent son 
geste. D'utilité évidente pour l'approche du gibier, la faculté mimétique leur permet de s'intégrer facilement à tous les milieux, d'osciller sans heurt entre des «contrasting forms of political, religious, geographical, cultural, and communicative idioms : state and segmentary, Muslim and pagan, urban and rural, modern and traditional, literate and oral, respectively " (p. 219). C'est donc grâce à la mimesis qu'ils négocient avec succès leur place entre l'islam, l'État et les communautés rurales. À l'instar de Manimori qui, en se dispersant dans la forêt, l'aurait alors maîtrisée d'une certaine manière (seulement laquelle ?), les chasseurs, en marchant sur ses pas, contrôleraient alors plusieurs domaines. En bref, selon l'auteur, le sacrifice instaurant le principe de réciprocité ainsi que la faculté mimétique permettant la maîtrise du monde représentent les deux tenants de la tradition cynégétique, deux raisons de l'efficacité des chasseurs et de leur constante popularité.

L'idée que les chasseurs soient particulièrement doués dans l'art de la mimesis ne surprend guère, Hellweg semble rejoindre ici Willerslev (2007) ${ }^{29}$. Mais lorsque ce dernier s'applique à disséquer les mécanismes concrets de cet $\operatorname{art}^{30}$, Hellweg reste quant à lui peu disert. Pour toute explication technique, il se contente de citer une formule magique comparant le chasseur au caméléon manipulant sa propre apparence. Qu'une telle manipulation de l'image puisse procurer le pouvoir, nous le savons bien: de nombreuses études démontrent que l'acte de copier quelque chose, c'est-à-dire de créer son double matériel ou graphique, est souvent une manière de s'approprier ce qui est imité. C'est la conclusion de Michael Taussig (1993) ${ }^{31}$ dont Hellweg reprend à son compte la théorie. Taussig insiste sur la présence de la magie mimétique au sein de l'État contemporain. Hellweg en convient lorsqu'il observe que les chasseurs, connus pour leurs savoirs en matière de métamorphose, imitent l'État ivoirien, dont le pouvoir se fonde sur la création d'un duplicata du pouvoir de l'État colonial ou précolonial, se référant lui aussi à un modèle originel. Ce qui nous semble particulièrement intéressant chez Hellweg, c'est justement la mise en dialogue des avancées de Taussig avec une vision compréhensive de la pratique et de la ritualité cynégétiques pour appréhender un phénomène concret - le Benkadi ivoirien - dans son historicité.

11 Toutefois, certains autres aspects de son ouvrage posent problème. Notamment la place réservée à la confrontation de matériaux ivoiriens de l'auteur avec les recherches sur les chasseurs dans d'autres pays de l'aire culturelle mandingue ainsi que celles concernant le contexte historique et religieux de leur activité. Ainsi, étrange nous paraît l'absence de référence aux travaux de Jean Bazin sur le pouvoir au sein de l'État précolonial mandingue ainsi qu'à des sources consacrées aux pratiques rituelles cynégétiques, pourtant relativement homogènes dans l'ensemble de l'aire culturelle envisagée (Kedzierska Manzon 2005, 2009, 2014) ${ }^{32}$. Il semble aussi curieux que les pratiques rituelles des chasseurs ne soient pas mises en rapport avec celles d'autres experts du religieux, tels que les officiants des cultes collectifs ou les détenteurs d'objets forts. Ceci étonne d'autant plus que ces deux types de pratiques possèdent de très nombreux points communs, comme le suggèrent les études, que Hellweg ne mobilise pas, portant sur la religiosité mandingue (Bazin 2008; Colleyn 2001, 2004, 2009; Kedzierska-Manzon 2013) ${ }^{33}$. Pourquoi alors l'auteur ne contextualise pas ses observations et ses données? Suppose-t-il que son terrain diffère tant du reste de l'aire culturelle en question que de telles références ne peuvent rien lui apporter? Ou bien ne considère-t-il pas que la ritualité cynégétique relève pleinement du champ religieux mandingue? Quoi qu'il en soit, son apport à la compréhension des dynamiques de pouvoir en Afrique de l'Ouest et sa conception novatrice du rôle de la littérature orale 
(cynégétique) qu'il interprète comme un «appel à l'action » font de son ouvrage une lecture à recommander.

\section{NOTES}

25. Y. T. CISSÉ, La confrérie des chasseurs malinké et bambara: mythes, rites et récits initiatiques, Yvry sur Seine, ACCT, 1994.

26. Hellweg définit ethos comme « a cultural logic underlying contrasting practices that orients practitioners towards analogous moral ends in pursuing these practices » (p. 226).

27. J. HELLWEG, « Hunters, Ritual and Freedom : Dozo Sacrifice as a Technology of Self in the Benkadi Movement of Côte d'Ivoire ", Journal of the Royal Anthropological Institute, 15 (1), 2009, pp. 36-56.

28. Il va de soi qu'Hellweg interprète le crime comme une entorse à la réciprocité.

29. R. WILLERSLEV, Soul Hunter: Hunting, Animism, and Personhood among the Siberian Yukaghirs, Berkeley, University of California Press, 2007.

30. Il insiste en l'occurrence sur les techniques du corps maîtrisées par les chasseurs et consistant en une imitation des mouvements des animaux, sur leurs costumes et d'autres accessoires, etc.

31. M. TAUSSIG, Mimesis and Alterity: A Particular History of the Senses, New York, Routledge ; London, Chapman \& Hill, 1993.

32. A. KEDZIERSKA MANZON, " "L'envol du vautour" : parole, action et objet dans les rituels funéraires des chasseurs malinké ", in U. BAUMGARDT \& F. UGOCHUKWU (dir.), Approches littéraires de l'oralité africaine, Paris, Karthala, 2005 ; «L'amour de la brousse : le rôle de l'espace dans la construction de l'identité du chasseur malinké », Journal des Africanistes, 79 (2), 2009 ; Chasseurs mandingues : violence, pouvoir et religion en Afrique de l'Ouest, Paris, Karthala, 2014.

33. J. BAZIN, Des clous dans la Joconde, Toulouse, Anacharsis, 2008 ; J.-P. COLLEYN, Bamana : the Art of Existence in Mali, New York, Museum for African Art, 2001 ; « L'alliance, le dieu, l'objet ", L'Homme, 170, 2004, pp. 61-75; «Images, signes, fétiches : à propos de l'art bamana (Mali)», Cahiers d'Études africaines, XLIX (3), 195, 2009, pp. 733-745; A. KEDZIERSKA MANZON, "Humans and Things: Mande "Fetishes" as Subjects", Anthropological Quarterly, 2013, pp. 1119-1151. 